In Crescendo. Institucional. 2016; 7(1): 11-20

Fecha de recepción: 6 de mayo de 2016

Fecha de aceptación: 17 de mayo de 2016

\title{
APLiCACIÓN DE LA OZONOTERAPIA EN PACIENTES CON ARTRITIS REUMATOIDE
}

\section{APPLICATION OF OZONE THERAPY IN PATIENTS WITH RHEUMATOID ARTHRITIS}

María Dolores Mondéjar Barrios* Lorenzo Orlando Rosas Durand**

\section{RESUMEN}

\begin{abstract}
bjetivo: evaluar la efectividad de la ozonoterapia en pacientes con Artritis reumatoidea. Metodología: se desarrolló un estudio de intervención preexperimental, antes y después, en 20 pacientes con diagnóstico de artritis reumatoidea, de mayo a octubre del 2014, que cumplieran con los criterios de inclusión establecidos. Se aplicó ozonoterapia por insuflación rectal combinada con autohemoterapia menor; las variables fueron el dolor, que se evaluó por la escala de graduación numérica, y la inflamación a través de los resultados de VSG y PCR. Resultados: el mayor por ciento de 45 a 49 años, predominó el sexo femenino, el factor de riesgo principal fue el sobrepeso. Se obtuvo respuesta satisfactoria en el $90 \%$ de los casos. Conclusiones: La ozonoterapia constituye una alternativa de tratamiento para la artritis reumatoidea; mejora el dolor y la inflamación, lo que justifica su uso, además de reforzar el sistema inmunológico, mejorando así la calidad de vida de los pacientes.
\end{abstract}

Palabras clave: artritis reumatoide, ozonoterapia.

\section{ABSTRACT}

Objective: To evaluate the effectiveness of ozone therapy in patients with rheumatoid arthritis. Methodology: In a pre-experimental pre-post intervention was developed in

\footnotetext{
* Médico. Especialista en Medicina Interna. Máster en Urgencias Médicas. Diplomada en Medicina Natural y Tradicional. Profesora. Investigadora. Docente de la escuela profesional Farmacia y Bioqímica - ULADECH

* Licenciado en Medicina Alternativa. Químico- Farmacéutico. Doctor en Ciencias Médicas. Especialización en ozonoterapia.
} 
20 patients diagnosed with rheumatoid arthritis, from May to October 2014, which met the inclusion criteria. It was applied ozone therapy for rectal insufflation combined with minor autohemotherapy; the variables were pain was evaluated by numerical scale Graduation and inflammation through the results of ESR and CRP. Results: the highest percentage of 45 to 49 years, females predominated; the main risk factor was overweight. satisfactory response in $90 \%$ of cases was obtained. Conclusions: The ozone therapy is an alternative treatment for rheumatoid arthritis; the improvement in pain and inflammation justifies its use, besides strengthening the immune system, thereby improving the quality of life of patients.

Keywords: Ozone therapy, rheumatoid arthritis.

\section{INTRODUCCIÓN}

La artritis reumatoide es una enfermedad crónica que afecta severamente la supervivencia, capacidad funcional y calidad de vida del individuo que la padece. Según estudios epidemiológicos realizados en los últimos 10 años, la artritis reumatoide causa una gran morbilidad, dificultad laboral e incremento de la mortalidad.

Esta enfermedad tiene una prevalencia en la mayoría de los países de 0,5\% a $1 \%$ de la población general. En Cuba se desarrolló el estudio epidemiológico COPCORD en el municipio Lawton, el cual le dio continuidad al estudio desarrollado a modo de pilotaje en el Cerro. Este trabajo permitió establecer las tasas de prevalencia y la discapacidad asociada para las diferentes enfermedades reumáticas. La prevalencia de la artritis reumatoide quedó fijada en $1.24 \%$ (1).

En Perú, Gamboa et al. reportaron un 0,5\% de prevalencia de artritis reumatoide. Es una enfermedad autoinmune, crónica, sistémica, y se manifiesta de forma variable, desde las molestias en las articulaciones hasta la incapacidad total, acompañada de dolores y en algunos casos de deformaciones. Puede ser extraarticular y causar daños en cartílagos, huesos, tendones y ligamentos de las articulaciones; además puede afectar diversos órganos y sistemas, como ojos, pulmones y pleura, corazón, pericardio, piel o vasos sanguíneos $(2,3)$.

El tratamiento convencional con fármacos produce varios efectos adversos y algunos de ellos serios, como es el caso del metrotexate como agente modificador de la enfermedad; con su uso debe vigilarse la función hepática (4).

La ozonoterapia es una alternativa, lo cual ayuda a disminuir las dosis de medicamentos al aliviar el dolor y la inflamación, además de elevar la inmunidad y mejorar la calidad de vida del paciente (5).

La ozonoterapia consiste en la aplicación del gas ozono, con el objetivo de provocar una respuesta terapéutica a través de la activación del sistema antioxidante enzimático y otros mecanismos celulares de adaptación a la oxidación, además de mejorar la oxigenación de los tejidos (6-8). 
El ozono es un gas natural, cuya molécula está formada por tres átomos de oxígeno con diversas propiedades beneficiosas para todo el organismo. $(9,10)$

En la artritis reumatoidea, el ozono actúa como inmunomodulador, ya que ha sido considerado como agente inductor ideal de citocinas, factor de necrosis tumoral $\alpha$, interleucina $1 \beta$; interleucina. Su eficacia inmunoduladora hace posible su aplicación en las enfermedades autoinmunes. La acción inmunológica del ozono está dirigida sobre los monocitos y los linfocitos $\mathrm{T}$, los cuales una vez inducidos liberan pequeñas cantidades de todas las citocinas y de interferón gamma. La liberación de citocinas se realiza de forma endógena. Asimismo, se obtiene una acción antiinflamatoria debido a la disminución de la producción de prostaglandina actuando sobre el ácido araquidónico (ácido graso insaturado), acción analgésica y germicida (contra bacterias, virus y hongos,; todo ello como consecuencia de la mejora del metabolismo celular y al mejor aporte de oxígeno a las células (11-15). Se realizó un estudio de intervención preexperimental con el objetivo de evaluar la efectividad de la ozonoterapia en pacientes con artritis reumatoidea.

\section{MÉTODOS Y TÉCNICAS}

Se realizó un estudio de intervención preexperimental antes y después, en pacientes con diagnóstico de artritis reumatoidea, de mayo a octubre del 2014.

Se trabajó con un universo de 20 pacientes. Los datos fueron recogidos de las historias clínicas y fueron almacenados en una base de datos para su posterior revisión, validación y procesamiento.

Como medida de resumen de la información se utilizó el porcentaje (\%). Los resultados se presentaron en tablas, las que se analizaron para emitir las conclusiones, en correspondencia con los objetivos de la investigación. Las diferencias estadísticas se evaluaron por la prueba de medias para muestras dependientes y la desviación estándar. Se tuvo en consideración los principios bioéticos de respeto a las personas, la beneficencia, la no maleficencia, justicia y autonomía. Se solicitó el consentimiento informado.

Se tuvieron en cuenta los siguientes criterios de inclusión y exclusión:

\section{Criterios de inclusión}

- Pacientes con diagnóstico de artritis reumatoidea.

- Pacientes que hayan emitido el consentimiento informado para ser incluidos en la investigación.

\section{Criterios de exclusión}

- Pacientes con trombocitopenia.

- Los que llevan tratamiento anticoagulantes.

- Pacientes con hipertiroidismo descontrolado.

- Hipertensión arterial y cardiopatías descompensadas. 
- Estatus epiléptico.

- Anemia intensa.

\section{Operalización de variables}

Edad: 20-24 años.

25-29 años.

30-34 años.

35-39 años.

40-44 años.

45-49 años

50-54 años

55-59 años

60 y más

Sexo: Masculino.

Femenino.

Peso: $\mathrm{Kg}$.

Talla: $\mathrm{cm}$.

IMC: Peso en kg/ talla en m.

Para la evaluación del dolor se usó la escala de graduación numérica de 1-10:

1, 2, 3, 4: dolor ligero.

5, 6, 7: dolor moderado.

8, 9, 10: dolor intenso.

Evaluación global de la enfermedad: Se realizará una evaluación global de la enfermedad desde el punto de vista del médico (EGM) y otra desde el punto de vista del paciente (EGP).

Para su medición, se recomienda usar una escala visual analógica horizontal de $10 \mathrm{~cm}$ dividida mediante marcas verticales, en 10 segmentos iguales de $1 \mathrm{~cm}$. Las mediciones se acompañarán de descriptores numéricos del 0 al 10, con indicadores en los extremos que señalen "muy bien" (0) y "muy mal" (10).

Reactantes de fase aguda: Las pruebas de laboratorio incluirán los reactantes de fase aguda: VSG y PCR. El comportamiento de estos dos reactantes de fase aguda tiene una estrecha relación con la actividad inflamatoria de la enfermedad. 
Evaluación del número de articulaciones dolorosas y del número de articulaciones tumefactas: se realizará mediante métodos validados basados en el recuento de al menos 28 articulaciones.

\section{Respuesta al tratamiento:}

Respuesta satisfactoria: cumplimiento de los criterios ACR20, menos de 6 articulaciones tumefactas y ausencia de afectación que produzca pérdida intolerable de la capacidad funcional.

Respuesta insatisfactoria: no cumplimiento de los criterios de respuesta satisfactoria.

\section{Los criterios ACR20:}

- Mejoría igual o superior al $20 \%$ en el recuento de articulaciones dolorosas y en el recuento de articulaciones tumefactas.

- Mejoría igual o superior al 20\% en al menos 3 de los siguientes parámetros: VSG o PCR, valoración global de la actividad de la enfermedad por el médico, valoración global de la actividad de la enfermedad por el paciente, valoración del paciente sobre el grado de dolor, discapacidad física.

Después de un minucioso interrogatorio y examen físico, se indicó hemograma completo y conteo de plaquetas, para descartarse las posibles contraindicaciones que pudiera tener el paciente para el tratamiento con ozono.

La insuflación rectal de ozono se realiza con una pequeña sonda, procede y para la autohemoterapia menor se extraen $10 \mathrm{ml}$ de sangre y esta se ozoniza a razón de $10 \mathrm{ml}$, concentración de $20 \mathrm{mg} / \mathrm{ml}$ y posteriormente se inyecta en el glúteo por vía intramuscular.

Se utilizó ozonoterapia por insuflación rectal en 20 sesiones divididas en 4 sesiones semanales a un volumen inicial de $100 \mathrm{ml}$ y concentración de $25 \mu \mathrm{g} / \mathrm{ml}$, de forma escalonada, se fue subiendo la dosis sin pasar de $200 \mathrm{ml}$ de volumen, ni concentración de $40 \mu \mathrm{g} / \mathrm{ml}$; se combinó con la autohemoterapia menor 1 vez a la semana hasta 6 sesiones. Al concluir el tratamiento se volvió a evaluar el paciente.

\section{RESULTADOS Y DISCUSIÓN}

Se realizó el tratamiento con ozono por vía rectal 20 sesiones (4 sesiones semanales) combinado con la autohemoterapia menor de 6 sesiones ( 1 sesión semanal) a 20 pacientes con el diagnóstico de artritis reumatoidea con los siguientes resultados (véase Tabla 1):

En la Tabla 1 se muestra la distribución de los pacientes de acuerdo a grupos de edad, predominando los de 45 a 49 años en un $30 \%$. Esta enfermedad se desarrolla con más frecuencia entre la $4 .^{a}$ y la $5 .^{a}$ década de vida, aunque puede afectar a cualquiera edad (16). 
Tabla 1. Distribución de los pacientes con artritis reumatoidea de acuerdo con grupos de edad.

\begin{tabular}{ccc}
\hline Grupos de edad & N. ${ }^{\circ}$ & $\%$ \\
\hline 20-24 años & 0 & 0.0 \\
$25-29$ años & 1 & 5.0 \\
30 -34 años & 1 & 5.0 \\
$35-39$ años & 0 & 0.0 \\
$40-44$ años & 1 & 5.0 \\
$45-49$ años & 6 & 30.0 \\
$50-54$ años & 5 & 25.0 \\
$55-59$ años & 3 & 15.0 \\
60 y más & 3 & 15.0 \\
Total & 20 & 100.0 \\
\hline
\end{tabular}

Tabla 2. Distribución de pacientes con Artritis reumatoidea según sexo.

\begin{tabular}{lcc}
\hline \multicolumn{1}{c}{ Sexo } & N. $^{\circ}$ & $\%$ \\
\hline Masculino & 1 & 5.0 \\
Femenino & 19 & 95.0 \\
Total & 20 & 100.0 \\
\hline
\end{tabular}

La Tabla 2 hace referencia a la distribución de pacientes en cuanto al sexo, siendo el sexo femenino el que prevaleció en un $95.0 \%$ de ellos. Es mayor la prevalencia de esta enfermedad en mujeres que en hombres, en relación 3:1, por lo general cuando comienza antes de los 60 años, ya que a partir de esta edad la relación hombre mujer se iguala (17).

Tabla 3. Distribución de pacientes con respecto a factores de riesgo modificables asociados.

\begin{tabular}{lcc}
\hline Factores de riesgo & N. $^{\circ}$ & $\%$ \\
\hline Sobrepeso & 13 & 65.0 \\
Tabaquismo & 2 & 10.0 \\
Estrés & 5 & 25.0 \\
\hline
\end{tabular}

En la Tabla 3 se observan los pacientes con respecto a los factores de riesgo que poseen, con un predominio del sobrepeso en el $65 \%$ de los pacientes. En mujeres jóvenes, el hecho de mantenerse con sobrepeso u obesidad durante más de 10 años aumenta el riesgo de padecer de artritis reumatoidea. Las personas con obesidad tienen menos probabilidad de responder al tratamiento con antinflamatorios esteroideos y AINE; la obesidad reduce la probabilidad de alcanzar la remisión en el tratamiento de la enfermedad con mayor presencia e intensidad del dolor articular. 
El tabaquismo afecta la respuesta inmune celular y humoral. Tiene efectos proinflamatorios como inmunosupresores a través de mecanismos diversos. El humo del tabaco contiene altas concentraciones de radicales libres y puede, además, aumentar la generación y activación de radicales libres endógenos. El consumo del tabaco aumenta la tasa metabólica basal y podría asociarse con una refractariedad al tratamiento antirreumático por interacciones farmacocinéticas o farmacodinámicas.

El estrés se ha relacionado con la artritis reumatoidea, pues los pacientes refieren con frecuencia episodios de estrés coincidiendo con el inicio de la artritis reumatoidea (18).

Tabla 4. Distribución de pacientes con artritis reumatoidea en cuanto a los resultados del tratamiento.

\begin{tabular}{lcc}
\hline \multicolumn{1}{c}{ Resultados } & N. ${ }^{\circ}$ & $\%$ \\
\hline $\begin{array}{l}\text { Desaparición del dolor y la tumefacción de las ar- } \\
\text { ticulaciones }\end{array}$ & 6 & 30.0 \\
$\begin{array}{l}\text { Mejoría en un 20\% o superior del dolor, VSG, PCR, } \\
\text { valoración global de la enfermedad y menos de 6 } \\
\text { articulaciones tumefactas. }\end{array}$ & 12 & 60.0 \\
$\begin{array}{l}\text { Mejoría en menos del 20\% del dolor, VSG, PCR, } \\
\text { valoración global de la enfermedad, más de 6 arti- } \\
\text { culaciones tumefactas ó ninguna mejoría. }\end{array}$ & 2 & 10.0 \\
\begin{tabular}{l} 
Total \\
\hline
\end{tabular} & 20 & 100.0 \\
\hline
\end{tabular}

La Tabla 4 refleja que en el $90 \%$ de los afectados con esta enfermedad se alcanzaron resultados satisfactorios con mejoría del dolor y de la inflamación.

El ozono médico se puede usar como un fármaco más con actividad reguladora general, que ejerce sus efectos al estabilizar el equilibrio redox celular. Los POL y el $\mathrm{H}_{2} \mathrm{O}_{2}$, generados por la descomposición del $\mathrm{O}_{3}$, se comportan como moléculas señalizadoras de estrés, lo que mejora el equilibrio energético celular y el sistema inmune en beneficio de enfermedades como son la psoriasis, el asma y la artritis reumatoide. Es considerado por diversos autores como una droga capaz de modificar la respuesta biológica por la multiplicidad de acción y la generación de señalizaciones intermoleculares (19-21).

Menéndez et al. añadieron satisfactoriamente, a lo largo de 8 semanas, una serie de inyecciones intramusculares de oxígeno/ozono en 17 pacientes tratados con antiinflamatorios no esteroideos (22). El mismo grupo de investigación en 2010 comparó las infiltraciones aisladas de $\mathrm{O}_{2} / \mathrm{O}_{3}(3 \mathrm{ml}$ a $10 \mu \mathrm{g} / \mathrm{ml})$ con las infiltraciones más ozonoterapia sistémica rectal en dos grupos de pacientes con artritis reumatoide de la articulación temporomandibular; las mejorías, tanto en el dolor, como en la función y en el estado de la cápsula articular fueron estadísticamente significativas a favor de la terapia combinada (23). A este respecto, la ozonoterapia sistémica parece disminuir los niveles de interleukina 1 beta (24), directamente relacionados con la actividad de la enfermedad, mientras que la ozonoterapia intraarticular disminuiría los niveles de interleukina 8 dentro de la articulación $(25,26)$. 
La ozonoterapia constituye una alternativa de tratamiento para la Artritis reumatoidea; la mejoría en el dolor y de la inflamación justifica su uso en esta enfermedad, además refuerza el sistema inmunológico y no provoca efectos adversos, mejorando así la calidad de vida de los pacientes.

\section{REFERENCIAS BIBLIOGRÁFICAS}

1. Guibert Toledano M, Reyes Gil A; Artritis reumatoide temprana. Retos y enfoque en el nuevo siglo. Revista Cubana de Reumatología 7-8; Vol. VII, 2005, 4 -7.

2. Krieckaert Charlotte LM, Willem F. Lems. Biologicals and bone loss. Therapeutic Advances in Musculoskeletal Disease. Ther Adv Musculoskel Dis. 2012; 4(4): 245-7. 3 .

3. Deal C. Bone loss in rheumatoid arthritis: systemic, periarticular, and focal. Curr Rheumatol Rep [Intenet]. 2012 [citado 23 octubre 2014]; 14:231-7. Disponible en: http://link.springer.com/article/10.1007/s11926-012-0253- 7\#page-1 4.

4. Thurah AD, Nørgaard M, Stengaard-pedersen IHK. Compliance with methotrexate treatment in patients with rheumatoid arthritis? inXuence of patients' beliefs about the medicine. A prospective cohort study. J Clin Epidemiol. 2010; 30:1441-8.

5. Schwartz Adriana, Claudia Nikolaevna Kontorschikova, Oleg Vitorovich Malesnoko, Gregorio Martínez Sánchez, Lamberto Re, Irina Avenerovna Gribkova. Guía para el uso médico del ozono - Fundamentos terapéuticos e indicaciones, AEPROMO, 2011, 315 p. ISBN: 978-84- 615-2244-6.

6. AEPROMO. Declaración de Madrid sobre la Ozonoterapia, Madrid, 2010, http:// www.aepromo.org/declaracion_madrid/Declaracion_madrid.pdf.

7. Pressman S y Warburg OH. The story of ozone. 6th ed. Plasmafire; 2000.

8. Ajamieh HH, Berlanga J, Merino N, Martínez-Sánchez G, Popov I, Menéndez S, Giuliano A, Re L, León OS. Role of protein synthesis in the protection conferred by ozone-oxidative-preconditioning in hepatic ischaemia/reperfusion. Transpl Int 2005; 18:1-9.

9. Barber E, Menéndez S, Barber MO, Merino N, Calunga JL. Estudio renal funcional y morfológico en riñones de ratas pretratadas con ozono y sometidas a isquemia caliente. Revista CENIC Ciencias Biológicas 1998; 29:178-81.

10. León OS. Effects of ozone oxidative preconditioning on nitric oxide generation and cellular redox balance in a rat model of hepatic ischaemia-reperfusion. Liver Int 2004; 24:55-62.

11. Menéndez S, et al. Ozono. Aspectos básicos y aplicaciones clínicas. Centro de Investigaciones del Ozono CENIC 2008; Ciudad de La Habana, Cuba. 
12. Carmona L. Revisión sistemática: Ozonoterapia en enfermedades reumáticas. Reumatol Clin 2006; 2:119-23.

13. Menéndez S. Mecanismos de acción biológica y efectos terapéuticos del ozono. En: Menéndez S, González R, Ladea OE, Hernández F, León OS, Díaz M. Ozono: Aspectos Básicos y Aplicaciones Clínicas. 1ra Ed. La Habana: CENIC; 2008. p. 4-107.

14. Díaz J, Martín N, Menéndez CS. Evaluación de la actividad inmunomoduladora del ozono sobre los leucocitos: in vivo e in vitro. Vaccimonitor 2011; 20 (1): 223.

15. Díaz J, Sardiñas G, Menéndez S, Macías C. Efecto inmunomodulador de la ozonoterapia en niños con deficiencia en la inmunidad mediada por fagocitos. Mediciego 2012; Vol. 18 (1).

16. Reyes Medine EA, Nerey González W, Suárez Rodríguez BL, Eguey Mesa JL. Caracterización clínico- epidemiológica de los pacientes con artritis reumatoide. Hospital Universitario Arnaldo Milián Castro. Revista Cubana Reumatología. 2015; XVII (1). ISSN: 1817-5996.

17. Videla CA, Civit de Garitnani E. Enfoque actual de la artritis reumatoidea. Separata, 2000.5-35.

18. Ho RCM, Fu EHY, Chua ANC, Cheak AAC, Mak A. Clinical and psychosocial factors associated with depression and anxiety in Singaporean patients with rheumatoid arthritis. Int J Rheum Dis. 2011; 14:37-47.

19. Díaz J, Macías C, Menéndez S. Efecto modulador de la ozonoterapia sobre la actividad del sistema inmune. Rev cubana Hematol Inmunol 2013; vol.29 (2).

20. Centro de Investigaciones del Ozono. Compendio de estudios y aplicaciones del Ozono en Cuba (1980-2004) [CD-ROM]. La Habana: SOFTCAL-CENIC, 2005.

21. Díaz J, Parés Y, Risco G. Aplicación de la ozonoterapia en la deficiencia Selectiva de IgA. Revista Cubana Hematol Inmunol Hemoter. 2009; 25(supl):265-301.

22. Menéndez F, Díaz G, Menéndez S. Ozonoterapia en la artritis reumatoidea. Revista CENIC Ciencias Biológicas 1989; 20:144-51.

23. Méndez-Pérez I, Cerro-Montesino A, Cámbara-Peña R, Martínez-Godine J, Menéndez-Cepero S. Ozonoterapia sistémica e intra-articular en la artritis de la articulación temporomandibular por artritis reumatoide. Revista CENIC. Ciencias Biológicas 2010; 41:169-72.

24. Fahmy Z. Correlation of plasma interleukin 1 levels with disease activity in rheumatoid arthritis with and without ozone. Proceedings in 2nd International Symposium on Ozone Applications. Habana, Cuba 1997.

25. Eastgate JA, Symons JA, Wood NC, Grinlinton FM, di Giovine FS, Duff GW. Correlation of plasma interleukin 1 levels with disease activity in rheumatoid arthritis. Lancet 1988; 2:706-9. 
26. Fahmy Z. Inmunological effect of ozone (O2-O3) in rheumatic diseases. Proceedings of the Eleventh Ozone World Congress, Ozone in Medicine 1993; p M 2-1 to M 2-8. 